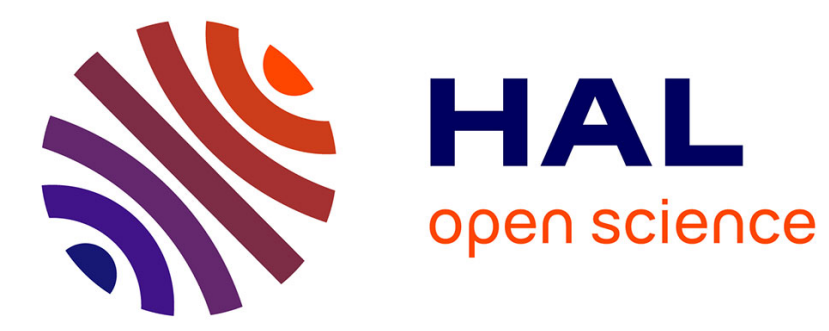

\title{
La fabrique de la rue en marche. Essai sur l'altération des ambiances urbaines
}

\author{
Jean-Paul Thibaud
}

\section{To cite this version:}

Jean-Paul Thibaud. La fabrique de la rue en marche. Essai sur l'altération des ambiances urbaines. Flux - Cahiers scientifiques internationaux Réseaux et territoires, 2007, 66, pp.111-119. 10.3917/flux.066.0111 . hal-00978341

\section{HAL Id: hal-00978341 \\ https://hal.science/hal-00978341}

Submitted on 11 Jul 2017

HAL is a multi-disciplinary open access archive for the deposit and dissemination of scientific research documents, whether they are published or not. The documents may come from teaching and research institutions in France or abroad, or from public or private research centers.
L'archive ouverte pluridisciplinaire HAL, est destinée au dépôt et à la diffusion de documents scientifiques de niveau recherche, publiés ou non, émanant des établissements d'enseignement et de recherche français ou étrangers, des laboratoires publics ou privés.

\section{(ㅇ)(1) $\$$}

Distributed under a Creative Commons Attribution - NonCommercial - NoDerivatives| 4.0 


\title{
La fabrique de la rue en marche: essai sur l'altération des ambiances urbaines
}

\author{
Jean-Paul Thibaud
}

\section{1- Qu'est-ce que peut un pas?}

En posant une telle question, il s'agit de donner droit de cité aux puissances de la marche et reconsidérer par là même nos façons de concevoir la rue. L'objectif est d'approcher la rue en train de se faire, dans sa dynamique de constitution et de transformation, à partir des mouvements qui l'animent, en tant qu'elle se fait et se défait au gré des flux piétonniers.

D'aucuns diront que la marche en ville devient de plus en plus négligeable au regard de la colonisation de la rue par l'automobile. Du point de vue de l'économie globale des flux urbains, elle relèverait d'un phénomène minoritaire ne portant pas à grandes conséquences. Et pourtant, dès que l'on prend au sérieux l'enjeu crucial que représente la rue pour la vie de la cité, la question de la marche ne manque pas de refaire surface. Il suffit de se demander ce que serait une rue sans piétons pour se convaincre du rôle primordial qu'ils jouent dans la vitalité de l'expérience urbaine. A l'heure où les mégapoles sont de plus en plus marquées par l'expansion indéfini des territoires, la prolifération des réseaux de toutes sortes et la recherche des grandes vitesses, la marche reste le garant de notre ancrage corporel et charnel à l'univers urbain. Bref, elle possède une valeur anthropologique fondamentale quant à notre manière d'habiter la ville. Comme le remarque à juste titre Olivier Mongin : «On ne se débarrasse pas, même au nom de la révolution globale en cours, du réel, du corps et du désir d'habiter dans un lieu, du corps à corps avec le monde ! »1.

Cela ne signifie pas pour autant que les pratiques piétonnières restent nécessairement identiques à elles-mêmes et soient préservées des mutations actuelles de la rue. Bien au contraire, étant particulièrement sensibles et réactives aux transformations de l'environnement urbain, elles en constituent un des principaux révélateurs. D'une certaine manière, en se demandant ce que peut un pas, il s'agit de mettre à jour ce que les rues d'aujourd'hui font à la marche et ce que celle-ci est susceptible de leur faire en retour. En d'autres termes, c'est le potentiel d'action et de transformation de la marche qui sera ici notre guide de lecture de l'évolution des rues. Pour questionner l'écologie sensible des flux urbains et clarifier le rapport entre la mise en marche des citadins et la mise en ambiance des rues, nous resterons au plus près de l'expérience quotidienne du passant en le suivant pas à pas et en l'observant au ras du sol. En procédant de la sorte, la rue sera conçue à la fois comme un champ de perception mobile et un domaine de fabrication dynamique, à l'articulation entre flux piétonniers et flux sensoriels.

\footnotetext{
${ }^{1}$ Mongin, O. La condition urbaine. Paris : Editions du Seuil, 2005, p. 229.
} 


\section{2- Pour mettre la rue en marche}

Nombreuses sont les approches qui ont cherché à mettre la rue en marche. Ainsi, on peut tenter d'expliquer pourquoi la marche est de plus en plus improbable en ville ou bien montrer comment l'aménagement matériel de la rue peut inciter à marcher. Dans cette perspective, la rue n'est pas réductible à une enveloppe neutre ou à un cadre inopérant, elle fonctionne plutôt comme un ensemble de ressources et de contraintes à la marche, variables selon les morphologies urbaines et les dispositifs construits. Première leçon : reconnaître à la rue son efficace pratique.

On peut aussi décrire comment les marcheurs urbains s'approprient la rue en la parcourant et en la récitant, comment ils la configurent au gré de leurs tours et de leurs détours. La marche est alors saisie en termes d'énonciations piétonnières et de rhétoriques cheminatoires qui confèrent une allure singulière à la rue. C'est ainsi que l'on ne s'intéresse pas seulement à ce qui fait marcher mais aussi aux manières de marcher, on ne prête pas uniquement attention à la fonctionnalité des rues mais aussi à leur physionomie et atmosphère particulières. Deuxième leçon : reconnaître à la rue son pouvoir d'expression.

On peut s'interroger par ailleurs sur la mise en forme des relations en public et observer comment les citadins agencent ensemble des situations de trafic. De ce point de vue, la marche en ville n'est en aucune façon une activité solitaire, elle mobilise nécessairement de petites attentions à autrui. La rue se présente alors comme le lieu de la civilité du pas, des échanges éphémères et des évitements passagers. Troisième leçon : reconnaître à la rue sa valeur d'urbanité.

On peut enfin chercher à dégager des figures du passant en montrant comment le rapport des citadins à la rue évolue selon les époques. Associé aux passages parisiens, le flâneur du début du XXème siècle constitue à cet égard une figure emblématique de la modernité. Dans cette perspective, la marche devient un révélateur des transformations de l'expérience en milieu urbain, de l'évolution des conduites perceptives en public et des mutations de la culture sensible de la grande ville. Quatrième leçon : reconnaître à la rue son enjeu socio-esthétique.

Qu'elle soit thématisée en termes d'action pratique ou d'expression habitante, d'interaction sociale ou d'histoire culturelle, la rue en marche est pensée dans tous les cas à partir de ce qu'elle effectue. Comme le montrent ces diverses approches, marcher ne se réduit pas à un simple déplacement d'un point à un autre, cela consiste aussi à détecter des informations de son entourage, configurer l'espace parcouru, modaliser son attention selon les circonstances, recadrer continuellement le paysage de la rue, mettre ses sens en éveil ou en veilleuse. Autant de façons de dire le caractère actif de la marche quant à la configuration sensible de la rue.

Mais encore, si la marche relève bien dans tous les cas d'une effectuation et d'un accomplissement, si elle se dévoile en fonction de ce qu'elle opère, ne peut-on pas tenter de l'appréhender aussi à partir de ce qu'elle génère ? 
En marchant, les passants ne sont pas seulement réceptifs aux émissions de la rue, ils en sont aussi des producteurs ; ils se donnent à voir et à entendre, émettent des signes et des signaux, s'échangent des propos et se racontent des histoires, se rendent eux-mêmes perceptibles et nuancent la rue de leur présence. De ce point de vue, la marche ne consiste pas seulement à être en prise ou en résonance avec l'environnement de la rue, elle est aussi un vecteur à part entière de sa fabrication. En reconnaissant la puissance d'engendrement de la marche, il s'agit de comprendre ce qu'elle fait à la rue et de déceler comment elle a - jusqu'à un certain point et dans certaines conditions - le pouvoir de l'altérer, de la moduler et de la transformer.

\section{3- Les ordres de grandeur de la marche}

Partons de l'hypothèse de la sensibilisation de la rue par la marche. Pour étayer cet argument, divers ordres de grandeur méritent d'être considérés. Quand nous avons affaire à des événements extraordinaires, relatifs à des marches un tant soit peu exceptionnelles, la mise en ambiance de la rue semble aller de soi. Pensons par exemple aux grands rituels de la vie sociale qui se déroulent en marchant : défilés militaires ou processions religieuses, cortèges politiques ou parades de carnaval... Tout un vocabulaire de la marche est ici décliné et mis à contribution, qui indique à la fois l'organisation collective des flux et la tonalité affective de l'événement. On peut aussi penser à certains rassemblements, plus ou moins fréquents, qui attirent et mobilisent les foules : marchés, braderies, brocantes, fêtes foraines ou fêtes de la musique... La marche devient ici piétinement et défilement ininterrompu. Ou bien alors, songeons aux divers spectacles de rue et actions artistiques qui entrainent parfois leurs publics dans des parcours urbains inusités : marches à plusieurs organisées par Fluxus, arpentages de territoires avec le groupe Stalker, pérégrinations à l'aide de géants démesurés avec Royal de Luxe... Que la marche se déroule au pas cadencé ou au rythme de danse, aux sons de fanfare ou de portes voix, qu'elle exprime un air de fête ou de mécontentement, elle devient le support principal à partir duquel la rue s'organise collectivement et se met en scène. Autant de collectifs mobiles qui la métamorphosent momentanément en l'investissant massivement et en prenant pour une fois le dessus sur le trafic routier. A haute teneur symbolique, souvent à l'échelle de la ville toute entière, ces marches hors du commun opèrent une véritable transfiguration de la rue, perçue et programmée comme telle.

Une autre façon de décrire les modalités de mise en ambiance de la rue est de prêter attention à la marche de tous les jours, banale, anodine, sans tambours ni trompettes. Il en va alors des trajets quotidiens qui participent de l'animation urbaine. Abraham Moles et Elisabeth Rohmer développent à cet égard une théorie originale des micro-événements pour rendre compte de la fabrique ordinaire de la rue. Chanteur de rue ou touriste demandant son chemin, sans domicile fixe en train de faire la manche ou enfant à la recherche de ses parents, interpellation d'un agent de police ou groupe de jeunes qui chahutent, militant distribuant un tract ou adeptes du skateboard s'entraînant à des figures, etc. Pas de phénomène massif ici mais une myriade 
de petits faits et gestes, incidents sans conséquence ou rencontres de hasard, anecdotes de toutes sortes qui ponctuent le parcours et retiennent l'attention des passants: "Tous ces petits faits de la rue entrent dans mon champ de conscience, sont acceptés et assimilés par lui, peut-être seront-ils retenus dans ma mémoire pour alimenter la conversation dans la sphère familiale. Les micro-événements sont les fibres du tissu du spectacle de la rue $»^{2}$. Les passants sont ici aussi bien acteurs que spectateurs, observateurs de ces scènes de rue impromptues tout autant qu'initiateurs involontaires de cellesci. De toute évidence, de nombreux traits distinguent les «marches extraordinaires » des «marches ordinaires". Les unes procèdent selon un même mouvement d'ensemble, de façon massive, organisée et souvent planifiée à l'avance ; les autres procèdent par micro-événements, selon de multiples orientations, sur un mode distribué et plus ou moins improvisé. Pour aussi différentes que soient ces deux formes d'investissement de la rue, elles ont néanmoins en commun de mettre en œuvre des phénomènes suffisamment saillants pour être un tant soit peu remarqués et remonter à la conscience des passants.

Il est une autre grandeur de la marche qui ne répond pas vraiment à cette condition, il s'agit de la marche « infra-ordinaire ». Celle-ci ne correspond pas à un troisième type de situation piétonnière, au même titre que les deux précédentes, mais bien plutôt à un changement de focale dans l'observation des phénomènes. Plus précisément, cette grandeur infra-ordinaire de la marche ne devient observable qu'au prix d'un travail de défocalisation de l'attention: "Au lieu du sens fixé, du signifiant distinct et des signes différenciés : l'attention flottante, la vision sans foyer, la vigilance éparse ${ }^{3}$. On se situe ici au seuil du remarquable, au niveau de ces états limites particulièrement difficiles à capter et à exprimer. Quoi de plus indicible mais aussi de plus immémorial - que le retentissement d'un talon sur le bitume ou le roulement d'un skateboard sur une rampe 4 , l'ombre mouvante d'un piéton sur la chaussée ou le reflet d'un passant sur un trottoir lavé par la pluie, l'empreinte d'un pas laissée dans la neige ou l'usure d'une dalle qui a finit par se patiner à force d'être piétinée ? Ancrage de la marche dans le sol. Affaire de surface quand la rue est foulée du pied : surface de diffusion sonore donnant à entendre les rythmes de la rue ${ }^{5}$, surface de projection lumineuse laissant entrevoir la «trame flottante de l'urbain $»^{6}$, surface d'inscription matérielle sédimentant des traces de passage. Encore faudrait-il préciser, partir à chaque fois de cas concrets, garder toujours à l'esprit que

\footnotetext{
2 Moles, A., Rohmer, E. Labyrinthes du vécu : l'Espace, matière d'actions. Paris: Librairie des Méridiens, 1982, p. 148.

${ }^{3}$ Galard, J. La beauté du geste. Pour une esthétique des conduites. Paris : Presses de l'Ecole Normale Supérieure, 1984, p. 76.

${ }^{4}$ Calogirou, C. \& Touché, M. Des jeunes et la rue : les rapports physiques et sonores des skateurs aux espaces urbains. Espaces et Sociétés. ${ }^{\circ}$ 90-91, 1997, p. 69-88.

${ }^{5}$ Lefebvre, H. Eléments de rythmanalyse. Introduction à la connaissance des rythmes. Paris : Editions Syllepse, 1992.

${ }^{6}$ Mons, A. L'ombre de la ville. Essai sur la photographie contemporaine. Paris: Les Editions de La Villette, 1994.
} 
« ce n'est jamais n'importe quel pied, ce n'est jamais n'importe quel sol ${ }^{7}$. Que dire également du souffle ténu de l'air déplacé en courant ou du sillage éphémère d'un parfum tracé en marchant, d'une bribe de conversation saisie au vol ou du frayage d'un piéton confronté à une foule, du rideau de passants qui n'arrêtent pas de défiler ou du pas de deux que tente un marcheur pour poursuivre sa course ? A l'ancrage de la marche dans la terre s'adjoint l'élan de la marche dans l'air. Il en va ici des dispositions et configurations sensibles d'un collectif en marche. Mais là encore il faudrait y regarder de plus près et spécifier concrètement l'agencement auquel on a affaire : côtoiement quand deux passants cheminent momentanément ou durablement l'un à côté de l'autre, défilement quand ils se rangent les uns derrière les autres, croisement quand ils se trouvent temporairement face-à-face... Entre la terre et l'air, la marche déploie mille et un presque riens qui confèrent à la rue sa texture sensible.

Bref, tout un ensemble hétérogène de gestes ambiants inhérents à l'acte de marcher et constitutifs de l'atmosphère d'une rue. D'une certaine manière, au registre des petites perceptions chères à Leibniz correspondrait celui des petites émissions, infimes souffles et influx minimaux de la rue issus des corps en mouvement. Sans doute pourrions-nous reprendre à notre compte la remarque de Roland Barthes concernant son expérience de la ville japonaise : «Recenser ces événements serait une entreprise sisyphéenne, car ils ne brillent qu'au moment où on les lit, dans l'écriture vive de la rue, et l'Occidental ne pourrait spontanément les dire qu'en les chargeant du sens même de sa distance : il faudrait précisément en faire des haïku, langage qui nous est refusé ${ }^{8}$.

\section{4- Le tournant sensible de la conception urbaine}

Avec l'idée de gestes ambiants c'est en fait toute l'écologie sensible de la rue qui peut être questionnée. Paradoxalement, c'est en prenant au sérieux le devenir de ces petites modulations sensibles de la rue qu'il semble possible de rendre compte de quelques tendances majeures la concernant. Dans les parties qui suivent, il s'agira de se demander ce que peut encore générer le pas d'un passant anonyme dans les rues d'une grande ville d'aujourd'hui. S'il est hors de propos d'élaborer ici une typologie exhaustive des rues à partir du traitement qu'elles réservent à la marche, on peut par contre mettre à jour quelques processus particulièrement symptomatiques de leur évolution actuelle. Mais commençons par dresser le décor.

Un trait notable de cette évolution relève précisément de l'intérêt croissant et explicite porté par les professionnels de l'urbain à l'environnement sensible des rues. De nombreux indices témoignent de cette tendance relativement récente: émergence de nouveaux domaines de compétence issus des métiers du spectacle et transférés à celui de la ville (scénographes urbains, designers sonores, concepteurs lumières...), développement d'instruments de gestion et de conception ad hoc (schémas

\footnotetext{
${ }^{7}$ Charles, D. Flux de marche avec piétinement. Traverses. $n^{\circ}$ 14-15, 1979, pp. 89.

${ }^{8}$ Barthes, R. L'empire des signes. Paris : Editions du Seuil, 2005, p. 109.
} 
directeurs d'aménagements lumineux, cartes de bruit et observatoires de l'environnement sonore, expérimentations lumineuses ou olfactives sur site, outils de modélisation et de simulation sonore, lumineuse ou thermoaéraulique...), interventions d'ordre esthétique sur des fragments urbains ordinaires et plus uniquement sur ceux présentant une valeur historique ou patrimoniale reconnue. Nous assistons manifestement à une nouvelle façon de concevoir la rue relevant de ce qu'il est désormais convenu d'appeler « l'urbanisme sensoriel »'. Le tableau ne serait pas complet si l'on n'intégrait également dans ce mouvement - et sans doute même au premier chef - les pratiques courantes de l'urbanisme commercial qui font elles aussi grand usage du contrôle de l'environnement à des fins marchandes ${ }^{10}$. Quoi qu'il en soit, il s'agit bien dans tous les cas de concevoir l'espace urbain en termes sensibles, à partir de ce qu'il donne à sentir et à percevoir. De la rue piétonne d'un centre historique à la galerie d'un centre commercial, du passage couvert à l'enclave ludique, du labyrinthe d'un espace souterrain à la promenade d'un parc de loisir, tout semble mis en œuvre pour maîtriser les ambiances et conserver une emprise optimale sur elles. Pierre Sansot s'inquiétait à ce propos de ce qu'il appelait la «frénésie paysagère ${ }^{11}$, de cette tentation de paysager toutes choses et tous lieux. La rue contemporaine se conçoit bel et bien désormais en termes de walkscape, dreamscape, soundscape et autres streetscapes.

La marche n'est pas absente de ce tournant sensible de la conception urbaine, bien au contraire, elle en constitue un fil directeur dans la mesure où les effets recherchés sont quasiment toujours conçus pour des citadins en mouvement. Comme le remarque Isaac Joseph: "Le regard que le scénographe porte sur l'espace urbain s'organise sur un découpage c'est-àdire sur une série de séquences ayant chacune leur ouverture et leur clôture. Mettre en scène l'espace l'urbain ce n'est donc pas l'apprêter pour un spectacle, faire qu'il en impose, c'est l'organiser sur un récit ou un parcours possible $\gg^{12}$. Puisque l'expérience vécue de l'environnement urbain s'effectue nécessairement en mouvement, c'est en partant de la mobilité des citadins qu'une conception sensible des rues devient possible. Mieux encore, les rues commencent à se distinguer les unes des autres quand on les considère à partir des gestes ambiants auxquels elles se prêtent. Prenez un simple pas et c'est en fait toute la contexture de la rue qui est convoquée.

\section{5- Des rues à l'épreuve des gestes ambiants}

Il nous faut maintenant arrêter de parler de la rue au singulier, comme catégorie générique, et tirer les conséquences de ce qui précède. Notons tout d'abord que l'acception que nous donnons au terme « rue » sera très large et très ouverte. Si la rue renvoie avant tout à un espace public découvert et

\footnotetext{
9 Zardini, M. (sous la direction de) Sensations urbaines. Une approche différente à l'urbanisme. Montréal : Centre Canadien d'Architecture, 2005.

${ }^{10}$ Sorkin, M. (Ed.) Variations on a Theme Park. New York : Hill and Wang, 1992.

${ }^{11}$ Sansot, P. Jardins publics. Paris : Editions Payot, 1993.

12 Joseph, I. L'espace public comme lieu de l'action. Les Annales de la Recherche Urbaine. $\mathrm{n}^{\circ}$ 57-58, 1992-1993, p. 213.
} 
orienté dans le sens de la longueur, nous verrons que de nombreuses variantes remettent en cause ces caractéristiques premières. Que devient donc la rue quand on la rapporte à de nouvelles formes d'espaces urbains aussi différentes que les centres commerciaux, les enclaves ludiques, les espaces souterrains, les parcs de loisirs, les atriums, etc. ? Autant d'espaces collectifs - mais la plupart du temps privés - accueillant du public et proposant des alternatives à la déambulation piétonne traditionnelle. De toute évidence, ces diverses propositions inventent et mettent en œuvre des morphologies urbaines originales. Pourtant, le propos n'est pas ici de prolonger une typomorphologie des rues contemporaines mais plutôt de mettre à jour - en s'appuyant sur des exemples et des descriptions - quelques processus de qualification les concernant.

Pour commencer, considérons le portrait que propose Richard Sennett de la $14^{\text {ème }}$ rue de Manhattan : «Les enfants apprennent à ajuster leur comportement : avec leurs yeux ils signalent aux autres qu'ils sont des amis, avec leurs corps ils évitent ceux qui ont l'air de chercher les ennuis. Ils ne peuvent y parvenir en partant, puisqu'ils doivent rester dans les parages de leurs parents ; cela suppose l'utilisation d'un code : l'endroit où l'on marche dans la rue, la façon dont on bouge ses bras... C'est une rue où les gens redéfinissent constamment leurs limites réciproques en recourant à leurs yeux, à leur corps. Ce genre de vie n'est possible que si l'on n'en parle pas, ce qui constitue un autre chevauchement : celui du dit et du non-dit. De plus, la vie sur la $14^{\text {ème }}$ Rue ne se règle pas en une seule fois, en un instant unique d'équilibrage. Les mutations sont constantes... ${ }^{13}$. Comme on le voit à travers cet exemple, une «rue vivante » n'est pas une rue figée dans le temps, immuable, mais au contraire une rue en mouvement, en devenir, accueillant une part irréductible d'indétermination. Dans un tel contexte, les gestes ambiants permettent de garder le sens et la mesure de l'autre : un pas ou un bras, des yeux ou un corps donnent le ton à l'ambiance de la rue en rendant sensible divers processus d'ajustement entre passants. La rue reste ici le domaine du contact avec autrui, du corps à corps négocié et de l'autorégulation tacite. Diverses variantes de ce type de rue pourraient être ici mentionnées. Annie Honnorat et Pierre Sansot ne disent pas vraiment autre chose à propos de ce qui constituerait une « rue gaie » quand ils remarquent qu'elle repose sur « une manière d'être au monde sans peser sur lui et aussi sans se laisser écraser par lui $\gg^{14}$. Là encore, il s'agit de développer un rapport équilibré entre le passant et son environnement. De son côté, Michèle Jolé montre bien que si une rue festive fonctionne comme une «matrice fusionnelle d'autocélébration », elle n'en repose pas moins sur une pluralité de publics ayant des «régimes d'engagement différents ${ }^{15}$. Dans ces exemples, on a affaire à des rues à caractère fondamentalement public et qui font de la marche un support privilégié du vivre-ensemble et du corps commun.

\footnotetext{
${ }^{13}$ Sennett, R. La ville à vue d'œil. Paris : Plan, 1992, p. 205.

14 Honnorat, A., Sansot, P. Qu'est-ce qu'une rue gaie? In L'esthétique de la rue. Textes réunis par Françoise Coblence, Sylvie Couderc et Boris Eizykman, Paris : L'Harmattan, 1998, p. 129.

15 Jolé, M. Le destin festif du canal Saint-Martin. Pouvoirs. $n^{\circ} 116$ «a rue », 2006, p. 127.
} 
Mais sitôt que l'on considère le devenir de la ville contemporaine, d'autres processus ne manquent pas d'émerger qui se combinent et s'interpénètrent pour transformer l'environnement sensible des rues et infléchir l'efficace des flux piétonniers. En prenant les gestes ambiants comme guide de lecture de ces mutations, nous lançons en quelque sorte des sondes afin de savoir comment la rue réagit aux pas des marcheurs. D'une certaine manière, il s'agit maintenant de procéder à une expérience de pensée consistant à radicaliser volontairement les logiques sous-jacentes et exagérer intentionnellement les processus en œuvre pour mieux les faire apparaître. En adoptant une telle attitude, nous nous inscrivons dans la lignée de Günther Anders pour lequel « il y a des phénomènes qu'il est impossible d'aborder sans les intensifier ni les grossir, des phénomènes qui [...] nous placent devant l'alternative suivante: 'ou l'exagération ou le renoncement à la connaissance' ${ }^{16}$. Bien sûr, ce que l'on propose ici n'a rien d'exhaustif et de définitif mais indique simplement quelques tendances remarquables affectant la qualité et la portée des flux piétonniers.

a- Contenir des pas. De plus en plus de rues à fonction essentiellement marchande tendent à limiter les puissances de la marche en la formatant. Dans ce cas, il est de moins en moins question de vagabonder au hasard et d'aller n'importe où sans but particulier. Plutôt que de laisser libre cours aux variations, improvisations et expressions du pas, il s'agit au contraire de le contenir, de le dompter, en restreignant autant que possible sa marge de manœuvre : « le corps est en jeu pour autant qu'est calculée l'emprise que l'on peut avoir sur lui $\gg^{17}$. Une des techniques les plus courantes consiste à canaliser les flux, baliser les parcours, inciter à circuler. L'exemple le plus évident et sans doute aussi le plus commenté est celui de Disneyland : «Disneyland est aussi le lieu de la passivité totale. Ses visiteurs doivent accepter d'y vivre comme ses automates : l'accès à chaque attraction est réglé par des mains courantes et des barrières de tubes métalliques, disposés en labyrinthe, qui découragent toute initiative individuelle ${ }^{18}$. En créant des couloirs de circulation piétonne, en démultipliant les barrières, potelets, chaînes et autres éléments de délimitation des territoires piétonniers, certaines rues de centre-ville ne sont pas complètement étrangères à cette propension à formater le pas des marcheurs. Les trajets sont alors prédéterminés, laissant peu de place aux chemins de traverse ou à la flânerie. En usant d'autres subterfuges, les centres commerciaux savent aussi particulièrement bien jouer de cette circulation induite pour transformer les visiteurs occasionnels en consommateurs captifs et désorientés ${ }^{19}$. Pour tout dire, la circulation tend ici à se substituer à la marche, les rues deviennent alors de purs espaces de circulation qui réduisent au maximum le nombre

\footnotetext{
${ }^{16}$ Anders, G. L'obsolescence de l'homme. Paris : Encyclopédie des nuisances, 2002, p. 29 sq.

${ }^{17}$ Ratouis, O. Le sens de la marche. Les Annales de la Recherche Urbaine. $\mathrm{n}^{\circ}$ 57-58, 1992-1993, p. 78.

${ }^{18}$ Eco, U. La Guerre du faux. Paris : Grasset, Le Livre de Poche, 1985, p. 73.

${ }^{19}$ Goss, J. The «Magic of the Mall »: An Analysis of Form, Function, and Meaning in the Contemporary Retail Built Environment. Annals of the Association of American Geographers. Vol. 83, n¹, 1993, p. 1847.
} 
d'actions possibles des passants et le répertoire des gestes ambiants à disposition.

b- Climatiser des espaces. L'histoire urbaine nous rappelle que la volonté de maîtrise de l'environnement des rues ne date pas d'hier. Que l'on se réfère aux rues à arcades ou aux passages couverts, divers dispositifs construits ont été inventés dans le passé pour contrôler certains facteurs climatiques. Avec les atriums, les galeries des centres commerciaux ou celles des espaces souterrains, nous assistons actuellement à un renforcement et une tentative de généralisation de ce processus. De plus en plus d'espaces apparentés aux rues se rangent désormais sous l'égide de l'« architecture capsulaire » : « La capsule est un dispositif qui crée une ambiance artificielle et qui, en formant son propre espace-temps sous forme d'un environnement (artificiellement) hermétique, minimise la communication avec l'extérieur $\gg^{20}$. Ces espaces enclos, introvertis et coupés du reste de la ville proposent un environnement sensible prédéfini dans lequel les gestes ambiants ne semblent pas vraiment de mise ou bien alors s'inscrivent dans une stratégie d'ensemble. Si l'on prend pour exemple les galeries d'un centre commercial, tout est à ce point conditionné et programmé que l'ambiance artificielle tend à se substituer, à recouvrir et à masquer si besoin est les petites émissions de la marche : «Les matières et lumières prédisposent à une atmosphère calme, à fond neutre, qui laissent le champ libre à l'accrochage visuel des enseignes commerciales et publicitaires. C'est à cette ambiance confortable, feutrée et tranquille que contribuent bien sûr le chauffage et la climatisation de l'air ambiant, tout autant que les éléments sonores standardisés de musique de fond dont les capacités relaxantes, émaillées d'informations stimulantes (messages publicitaires, ...), sont distillées par des firmes spécialisées $\gg^{21}$. Dans un tel environnement, la marche procède d'un état de quasi-apesanteur et convoque un sentiment de flottement. Elle s'inscrit dans une atmosphère irréelle et atemporelle dans laquelle le passant n'a que peu de prise.

c- Animer des publics. Nous n'avons sans doute pas insisté suffisamment jusqu'à présent sur la charge symbolique que recèlent certains gestes ambiants. Le retentissement d'un pas dans une rue déserte ou l'ombre déformée d'un rôdeur la nuit font désormais partie de ces archétypes de l'expérience urbaine si bien mis en scène au cinéma. Mais si ces images restent très ancrées dans l'imaginaire social des citadins, elles empruntent désormais d'autres formes et d'autres supports. Tout se passe comme si, pour être empruntées, les rues d'aujourd'hui devaient raconter des histoires toutes faites, accompagner la marche d'une intrigue connue d'avance, proposer un spectacle grandeur nature. Pensons par exemple aux parcours historiques ou audioguides pour touristes que proposent désormais la plupart des villes actuelles. Pensons aussi aux enclaves ludiques organisées « de manière à créer un ensemble festif, dense, animé, dans lequel le parcours du visiteur est censé s'inscrire dans une expérience globale lui apportant des sensations

\footnotetext{
${ }^{20}$ De Cauter, L. La civilisation capsulaire : la ville à l'ère du capitalisme transcendantal. Les Cahiers de la Cambre. $\mathrm{n}^{\circ} 1,2002$, p. 20.

${ }^{21}$ Martinez Jamart, M. Macro-malls, Micro-cities. Les Cahiers de la Cambre. $\mathrm{n}^{\circ} 1,2002$, p. 95.
} 
inédites ${ }^{22}$. Pensons à la plupart des galeries marchandes et autres espaces commerciaux qui offrent de plus en plus des promenades à thèmes. Le pas des visiteurs - mais nous devrions dire plutôt des consommateurs tant ces espaces sont orientés vers le commerce et le rapport marchand - est bel et bien soumis à une " politique de l'animation ${ }^{23}$ lui procurant des décors exotiques, nostalgiques ou futuristes. L'exemple le plus éclatant de cet essai de réenchantement du monde urbain réside probablement dans Las Vegas qui invente à sa manière un nouveau type de rue : « En décloisonnant le spectacle des salles et des parcs, les promoteurs de Las Vegas ont créé la rue-spectacle, la ville comme mass-médium ${ }^{24}$. Dans ce cas, la marche s'inscrit dans une écologie de la fantaisie laissant que peu de place à l'imprévu et bombardant le visiteur de stimulations de toutes sortes. Les passants deviennent ici davantage des spectateurs que des acteurs à part entière de la rue, la marche tend à devenir une simple composante de la réception du spectacle en cours.

$d$ - Effacer des traces. Une autre façon de poursuivre l'objectif d'un environnement idéal des rues consiste à l'épurer au maximum de ses supposées scories. Ainsi, avec les opérations de restauration ou projets de réhabilitation, on assiste à la mise en œuvre d'un « urbanisme clean » consistant à «faire place nette $»^{25}$. Dans ce cas, il s'agit de faire le vide, de gommer la patine du temps, d'effacer les traces d'usage. Bref, la rue tend ici à être refaite à zéro, comme si rien ne s'était vraiment passé qui mérite que l'on s'en souvienne et que l'on garde en mémoire. Dans les centres historiques anciens, on est pris dans ce double mouvement de patrimonialisation d'une part (la rue se rapproche parfois d'un véritable musée commémoratif des grands événements du passé) et de stérilisation d'autre part (la rue se rapproche alors d'un pur décor reconstruit de toutes pièces). Dans un tel contexte, la marche perd de sa capacité à s'inscrire à même le sol et à marquer la rue de son empreinte. Les traces qu'elle laisse ou qu'elle pourrait laisser ont tendance à disparaître aussitôt. Cette politique du « grand nettoyage » va dans certains cas si loin qu'il est parfois nécessaire de réintroduire artificiellement des traces d'usage. Comme le relève Mike Davis à propos du Universal City Walk à Los Angeles, pour atténuer cette sensation d'artificialité, le concepteur « Jerde proposa d'ajouter une 'patine temporelle' et un 'soupçon de saleté' " à ce nouvel aménagement de rue ${ }^{26}$. Mais plus généralement, les substituts de rues que proposent les parcs de loisirs ou les centres commerciaux s'arrangent pour "s'affranchir des nuisances de la vraie ville $»^{27}$, que cela concerne l'univers routier (embouteillage, pollution, bruit) ou l'univers social (mendiants, SDF, groupes

\footnotetext{
${ }^{22}$ Gravari-Barbas, M. Les enclaves ludiques : Le cas du Navy Pier à Chicago. In Réinventer le sens de la ville. Sous la direction de Cynthia Ghorra-Gobin, Paris : L'Harmattan, 2001, p. 160.

${ }^{23}$ Hajer, M., Reijndorp, A. In Search of New Public Domain. Rotterdam : NAi Publishers, 2001.

${ }^{24}$ Bégout, B. Zéropolis. Paris : Editions Allia, 2002, p. 85.

${ }^{25}$ Dollé, J.-P. Le territoire du rien. Paris : Editions Lignes et Manifestes, 2005.

${ }^{26}$ Davis, M. Au-delà de Blade Runner. Los Angeles et l'imagination du désastre. Paris : Editions Allia, 2006, p. 87.

${ }^{27}$ Didier, S. Parcs de loisirs et nouveaux espaces publics. In Réinventer le sens de la ville. op. cit. p. 154.
} 
ou personnes «indésirables»). Dans cette recherche utopique de la perfection et de l'espace « idéal », la rue tend à devenir un espace lisse, sans accroche, sans frottement et sans tension, conduisant à un appauvrissement de l'expérience vécue des passants. Pour paraphraser Walter Benjamin, si habiter la rue consiste à laisser des traces en marchant, alors nous avons affaire ici à des propositions de rues difficilement habitables.

e- Détecter des présences. Dans le cas extrême de la «rue-fantôme » à laquelle est souvent associée Los Angeles, la rue a tendance à se dépeupler, à être désertée et vidée de son public. C'est la possibilité même d'être à pied qui est problématique tant cet état peut paraître incongru, inapproprié, voire suspect dans un univers de l'automobile et des freeways. Si l'on en croit les visions les plus alarmantes en la matière, une telle situation urbaine consacrerait le règne de l'écologie de la peur et de la surveillance généralisée. La mean street se transformerait en un « paysage virtuel » - un scanscape - et ne constituerait somme toute que l'image inversée des communautés encloses (gated communities). On pourrait croire au premier abord qu'un tel contexte n'a rien à faire et rien à apprendre de l'idée de geste ambiant. Cette notion serait déplacée et inappropriée et ne possèderait en tout cas ici aucune valeur heuristique. Mais quand on y regarde de plus près, on s'aperçoit que les gestes ambiants deviennent en fait un des objets privilégiés de la vigilance paranoïde en œuvre dans ces rues. Quand geste ambiant il y a, trace sensible d'une quelconque présence humaine, c'est dans les termes des systèmes de détections les plus sophistiqués « qui comprennent déjà dans leur éventail panoptique la vision, l'odorat, la sensibilité à la température et à l'humidité, les capteurs de mouvements et, dans certains cas, l'ouïe ${ }^{28}$. Dans le pire des cas, les petites émissions d'un corps en mouvement se transforment en instrument de gestion et de contrôle des populations.

\section{6- L'altération des ambiances de la rue}

La marche constitue sans doute l'activité élémentaire à partir de laquelle la rue se met à vibrer et prendre corps. Dans la vie de tous les jours, elle procède par petites touches et menues variations qui infusent l'environnement sensible des rues. Ces émissions infinitésimales - ce que nous avons appelé des gestes ambiants - ne se laissent pas aisément saisir, tant prédomine leur caractère diffus, éphémère et infraliminaire. Mais si l'on ne s'aperçoit pas toujours de ce qui nous affecte, cela ne signifie pas pour autant que nous y sommes insensibles. D'une certaine manière, on pourrait dire qu'un geste ambiant constitue l'unité élémentaire de la fabrique sensible d'une rue, le format de base à partir duquel les flux de la rue s'incarnent et se ressentent. Comme l'analyse si bien Tim Ingold : «En habitant le monde on n'agit pas sur lui, on ne fait pas des choses au monde; bien plutôt nous nous embarquons avec lui. Nos actions ne transforment pas le monde, elles sont

${ }^{28}$ Davis, M. op. cit. p. 26. 
une partie et une parcelle du monde en train de se transformer lui-même ${ }^{29}$. C'est bien de cela dont il s'agit ici, de la valeur d'imprégnation de la marche et de sa capacité à nuancer le milieu ambiant de la rue tout en se fondant à lui.

Ce propos très général ne prend toutefois véritablement de sens qu'à partir du moment où on le rapporte à des cas concrets, situés et datés historiquement. En effet, toutes les rues ne s'équivalent pas en matière de marche, elles peuvent avoir une emprise plus ou forte sur elle, être plus ou moins bien disposées à son égard, plus ou moins ouvertes aux variations sensibles qu'elle offre. C'est ainsi que l'on peut se demander ce qu'il en est encore de la valeur d'imprégnation de la marche dans les rues du XXI ${ }^{\text {eme }}$ siècle. Bref, à quelles conditions la marche parvient-elle à exercer pleinement sa capacité à générer - ou pour le moins nuancer - les ambiances d'une rue ? Les quelques processus identifiés et rapidement passés en revue ont permis de montrer que la mise en ambiance des rues semble être de plus en plus cadrée et formatée. Contenir des pas, climatiser des espaces, effacer des traces, animer des publics ou détecter des présences constitue autant de manières de conditionner l'environnement sensible des rues en réduisant la part d'indétermination inhérente aux activités ordinaires des passants. Faut-il voir ici une illustration de la société de contrôle telle qu'en ont parlé Michel Foucault et Gilles Deleuze au terme de leur vie ? Quoi qu'il en soit, si la morphologie construite des rues prend part à cette « gouvernance sensorielle des flux urbains ", c'est en se conjuguant aux systèmes techniques les plus sophistiqués (systèmes de climatisation, de circulation, de surveillance) et aux techniques d'animation contemporaines (son et lumière, espaces à thèmes, mise en récit des rues). En d'autres termes, la conception urbaine des rues ne convoque pas seulement leur stricte composante matérielle mais aussi de plus en plus sur leur face immatérielle (sensible, émotionnelle).

On l'aura compris, la lecture des rues que nous proposons tente de déchiffrer comment les mutations urbaines actuelles opèrent un nouveau «partage du sensible ${ }^{30}$ et occasionnent de nouveaux modes de sentir. Dans l'entre-deux guerres, Walter Benjamin et Siegfried Kracauer s'étaient saisis des rues pour mettre à jour l'esthétique du choc et la culture de la distraction à l'œuvre dans la ville moderne. Il semblerait que si de nombreux points de leurs analyses conservent toute leur actualité et restent prémonitoires à bien des égards, l'environnement sensible de la rue s'est néanmoins considérablement transformé depuis. Peut-être assistons nous maintenant à l'émergence d'une nouvelle forme de la sensibilité de l'ordre de l'anesthésie. Tout se passe comme si les flux de la rue favorisaient dorénavant l'expérience du flottement sur celle de la friction, privilégiaient le flot ininterrompu sur la modulation rythmique, composaient davantage par saturation que par imprégnation.

\footnotetext{
29 Ingold, T. The Temporality of the Landscape. World Archaeology. Vol. 25, n 2, October 1993, p. 164 (traduction personnelle de l'anglais).

${ }^{30}$ Rancière, J. Le partage du sensible. Paris : La Fabrique-éditions, 2000.
} 